\title{
Chronic fatigue syndrome and cognitive deficit are associated with acute-phase neuropsychiatric manifestations of COVID-19: A 9-month follow-up study
}

\author{
Fatemeh Sadat Mirfazeli ${ }^{1}\left({ }^{10} \cdot\right.$ Atiye Sarabi-Jamab $^{2}(\mathbb{D})$ Victor Pereira-Sanchez $^{3,4}\left(\mathbb{D} \cdot\right.$ Alireza Kordi $^{5}(\mathbb{D}$.

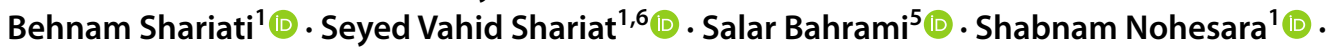 \\ Mostafa Almasi-Dooghaee ${ }^{7}$ (1) . Seyed Hamid Reza Faiz ${ }^{8}$ (D)
}

Received: 19 September 2021 / Accepted: 24 November 2021 / Published online: 21 January 2022

(c) Fondazione Società Italiana di Neurologia 2021

\begin{abstract}
The prevalence of long-COVID symptoms is rising but it is not still possible to predict which patients will present them, and which types of symptoms they will present. We followed up 95 patients with confirmed COVID-19 for 9 months to identify and characterize long-COVID symptoms. Easy fatigability was the most common symptom (51.04\%), followed by anxiety (38.54\%), dyspnea (38.54\%), and new-onset headache (38.54\%). There was no association between COVID-19 severity in the acute phase and the number of long-COVID symptoms $(F(1,93)=0.75, p=0.45)$, and cognitive function (MoCA) scores $(F(1,90)=0.073, p=0.787)$ at follow-up. Being female $(F(1,92)=-2.27, p=0.02)$, having a higher number of symptoms $(F(1,93)=2.76, p=0.0068)$, and experiencing constitutional neuropsychiatric symptoms $(F(1,93)=2.529, p=0.01)$ in the acute phase were associated with having chronic fatigue syndrome at follow-up. Moreover, constitutional neuropsychiatric symptoms in the acute phase were associated with a lower MoCA score $(F(1,93)=10.84, p=0.001)$ at follow-up. Specific clinical presentations such as constitutional neuropsychiatric symptoms in the acute phase might be predictors of debilitating long-COVID symptoms such as chronic fatigue syndrome and cognitive deficits.
\end{abstract}

Keywords COVID-19 $\cdot$ Long-COVID $\cdot$ Neuropsychiatric symptoms $\cdot$ Follow-up $\cdot$ Chronic fatigue syndrome

\section{Introduction}

As of this writing, more than 200 million cases of COVID19 have been reported worldwide, most of which have resulted in recovery [1]. COVID-19 is recognized as a multi-organ disease with a broad spectrum of manifestations, and there are increasing reports of long-term effects after acute COVID-19 [2]. Some authors include in the definition of post-acute sequelae of SARS-CoV-2 infection (PASC) symptoms lasting more than 3 weeks after onset of the disease, whereas chronic symptoms of SARS-CoV-2 (CSSC) or long-COVID would include those extending beyond 12 weeks $[3,4]$. The prevalence of PASC and CSSC

Fatemeh Sadat Mirfazeli and Atiye Sarabi-Jamab contributed equally to this work as first authors.

Seyed Hamid Reza Faiz

Shr.faiz.anesthesiology@gmail.com; hrfaiz@hotmail.com

Extended author information available on the last page of the article or long-COVID varies across studies, probably based on the severity of the acute symptoms, the duration of the followup, and other factors. Greenhalgh et al. reported a prevalence of $10 \%$ after 3 weeks [3]. In a post-acute COVID-19 study in the USA with a 60-day follow-up after discharge, it was found that $6.7 \%$ of patients died, $15.1 \%$ required readmission, and $32.6 \%$ of reported persistent or even new-onset symptoms [5]. In a 6-month follow-up study after discharge,76\% of the patients had at least one residual symptom (not counting the patients who died, were readmitted, had psychosis or dementia, or were physically immobile). In this study, fatigue (63\%), sleep difficulties (26\%), hair loss (22\%), and smell disorder (11\%) were the most frequent residual symptoms [6].

Like several other respiratory viruses, COVID-19 has a neuroinvasive potential. It may damage the central nervous system as a result of virus-induced neuro-immunopathology and/or virus-induced neuropathology. Other mechanisms of brain involvement may be mediated through the effect of the virus on other organs and systems (such as the liver, 
kidneys, and hypoxia) or through virus-induced coagulopathy. The psychosocial impact of the disease, with isolation and emotional distress, are other important concerns after the acute phase [7]. Therefore, it is not surprising that neuropsychiatric symptoms are among the most common post-acute symptoms. In one study, over $30 \%$ of hospitalized COVID-19 patients exhibited cognitive problems, depression, and anxiety, which persisted for months after discharge [7]. In other research reports, it was registered that 1 month after infection, there was a considerably high prevalence of insomnia (34-40\%), depressive symptoms (31-45\%), anxiety symptoms (22-47\%), post-traumatic stress disorder (28\%), and obsessive-compulsive symptoms $(20 \%)[8,9]$. In a survey of depressive symptoms, Perils et al. found a prevalence of $52.4 \%$ for these symptoms among COVID-19 patients, and that the presence of headache was associated with higher probability of moderate or more severe depressive symptoms [10].

Neurocognitive impairments are another category of post-COVID symptoms. In one study, 34\% of 279 hospitalized COVID-19 patients reported memory loss, and 28\% described impaired attention 3 months after discharge [11]. Other cognitive symptoms including impaired memory and receptive language and/or executive dysfunctions have been reported [12]. New-onset dementia after hospitalization for COVID-19 was shown to be 2-3 times more frequent than what was observed after hospitalization for other medical conditions [13].

However, it is not yet clear which patients will develop PACS and CSSC symptoms, specifically chronic neuropsychiatric symptoms, after infection with COVID-19. A few efforts have been carried out to find the association between clinical manifestations and sociodemographic factors of patients and neurocognitive sequelae of COVID-19. For example, Woo et al. studied 18 patients with mild to moderate COVID-19 disease with a mean age of 42 years and found that after around 85 days, over $75 \%$ of patients had difficulties in episodic memory, attention, and concentration, but these cognitive deficits were not associated with a history of fatigue, depression, hospitalization, treatment, viremia, or acute inflammation [14]. However, in other studies, delirium, as one of the most important neurocognitive symptoms during the acute phase of illness, was suggested to contribute to long-term cognitive decline $[15,16]$. Moreover, in other studies, post-COVID manifestations were reported to be related to comorbidities and disease severity [17]. Most of the literature on predictors of long-COVID symptoms is either subject to a small sample size [14] or contradicting results [15-17].

Therefore, in this study, we set to investigate the association of acute clinical manifestation of the COVID-19 and sociodemographic features of the patients, and the experience of future long-COVID symptoms. We further investigated whether the severity of acute clinical manifestation is a predictor of future long-COVID symptoms. We hypothesized that the pattern of long-COVID symptoms would not be random and those with more acute systemic and neuropsychiatric manifestations would have a higher probability to present with neuropsychiatric symptoms and chronic fatigue syndrome in the long term.

\section{Material and methods}

\section{Participants}

The present study was a 9-month follow-up of 201 participants with a diagnosis of COVID-19 (52 outpatients and 149 inpatients) from March 2019 to April 2020 in a large tertiary hospital (with which the authors are affiliated) in Tehran. The findings of the baseline assessments of these individuals during their acute COVID-19 phase are reported elsewhere [18]. COVID-19 diagnosis was based on a positive real-time reverse transcription-polymerase chain reaction assay using a COVID-19 nucleic acid detection kit or an abnormal chest computerized tomography scan compatible with a typical finding of COVID-19 as reported by three specialists (an emergency medicine specialist, an infectious disease specialist, and a pulmonologist). The diagnosis was supported by related laboratory tests (biochemistry and inflammatory factors). Patients were diagnosed with COVID-19 according to the World Health Organization interim guidelines [19].

From the 201 initial patients, 95 were retained through follow-up (median age $=50$ [range 28-86], 58\% male $[n=55])$. We follow patients for a median of 270 days (range $=258-300)$. Losses to follow-up included 8 deaths due to COVID-19 and 99 individuals who declined to participate or who were unresponsive to the invitation.

All patients gave informed consent for the study. This research was approved by the ethics committee of the Iran University of Medical Science (code IR.IUMS. REC.1399.1040).

\section{Data collection}

In the baseline study [18], patients with mild to moderate COVID-19 filled an online comprehensive symptom checklist regarding their acute phase, and a trained neurology resident examined patients with moderate to severe COVID-19 who were hospitalized. Patients' electronic paraclinical data such as imaging and laboratory tests were also incorporated.

In the current study, two trained medical students followed up with the patients via phone interviews. We also used the information about the clinical and paraclinical manifestation of the patients, drug history, comorbidity, and 
sociodemographic profile that was collected 9 months before during the baseline study.

\section{Symptom report}

We documented symptoms reported after recovery from COVID-19 using a comprehensive checklist of specific symptoms of different organs potentially correlated with COVID-19, drug history, demographic information, and comorbidity. We also registered chronic fatigue syndrome based on the Centers of Disease Control and Prevention criteria [20], which is a neurological disease characterized by severe mental and physical fatigue, chronic pain, and sleep disorder. Patients were asked to specifically remember symptoms that started after their diagnosis of COVID-19, those that started after recovery from COVID-19, and those which persisted.

\section{Cognitive assessment}

We used a Persian translated version of the Montreal Cognitive Assessment (MoCA)-BLIND 7.1 for telephonic cognitive assessment. MoCA is extensively used for the screening of mild neurocognitive impairment and evaluates a different aspects of cognitive abilities including orientation, shortterm memory/delayed recall, executive function, visuospatial ability, abstraction, naming, language, attention, and clock drawing test [21] (available at www.mocatest.org). A telephone-assessment version, devoid of its visual component, has been also validated [22-24]

\section{Statistical analysis}

All statistical analyses were performed using $\mathrm{R}$ version 3.6.1 (R Core Team, 2019) along with R Studio and packages "dplyr" and "rlang" for data manipulation, and also "ggplot2" for data visualization. For numerical variables, we used mean and standard deviation (SD) when they were normally distributed, and median and range if they were not. We used analysis of variance (ANOVA) to compare differences in means across groups. Correlations were tested using Pearson's correlation coefficient. To find the predictor factor of our dependent variables, we performed a (generalized) linear model and in the case of more than one predictor factor, we performed a stepwise regression by iteratively adding and removing variables in each step. To do this in $\mathrm{R}$, we used the "MASS" package, which chooses the best model based on the Akaike information criterion (AIC), and we used the "stepAIC" function for stepwise regression. Moreover, we used "rsq" package to calculate the R-squared and partial correlation coefficients for generalized linear (mixed) models. Statistical significance was set at a 2 -tailed $p$-value threshold of $<0.05$.

\section{Results}

\section{Description of long-COVID symptoms in the follow-up of patients}

In the descriptive analysis, easy fatigability (51.04\%) was found to be the most common long-COVID symptom, followed by anxiety (38.54\%), dyspnea (38.54\%), and new-onset headache (38.54\%). Eighty-six (90\%) patients showed at least one long-COVID symptom in their followup. Figure 1 displays the frequency of all the symptoms reported by our cohort of patients.

Persistent symptoms were then clustered based on their similarity of symptoms and the clusters identified in our previous study with acute-phase symptoms [18]. We obtained 8 main clusters: constitutional neuropsychiatric symptoms (palpitation, dizziness/vertigo, anxiety, mood swings, irritability, new-onset suicidal ideation, paresthesia, depression), dermatologic symptoms (skin rash, newonset hair loss), respiratory symptoms (post-exertional dyspnea, constant dyspnea, occasional dyspnea), gastrointestinal symptoms (constantdiarrhea/constipation, constant stomachache, low appetite), chronic fatigue syndrome (sore throat, new-onset headache, constant muscle pain, constant joint pain, easy fatigability, constant fatigue, constant weakness, impaired concentration/forgetfulness, unrestful sleep, tender lymphnodes), specific neuropsychiatric symptoms (auditory hallucinations, visual hallucinations, vision impairments, visual distortion, double vision), and ear-nose-throat symptoms (constant hearing loss, tinnitus). Constitutional neuropsychiatric symptoms were the most commonly reported cluster of symptoms (58.33\%), as shown in Fig. 2.

Generalized linear regression analysis exploring sociodemographic predictors of chronic fatigue syndrome indicated that gender was a predictor of chronic fatigue syndrome $($ Beta $($ Male $)=0.195, p=0.02)$. Age was not a significant predictor $($ Beta $=0.004)$. The overall model fit was $R^{2}=0.073$. As depicted in Fig. 3, the ANOVA analysis showed that this syndrome was more common in female patients: $64 \%$ female (median age $=49$ [range $36-86$ ) ; 36\% male (median age $=48.5$ [range 39-77]; $F$ $(1,93)=5.654, \mathrm{MSE}=23.20, p=0.02)$.

\section{Relation between clinical manifestations in the acute phase of COVID-19 and long-COVID symptoms}

In our acute-phase study [18], we had categorized patients' acute-phase symptoms in six clusters: constitutional-like neuropsychiatric (including dizziness, headache, and limb 

long-COVID symptoms in the follow-up of 95 patients with a history of COVID-19
Fig. 1 The frequency of

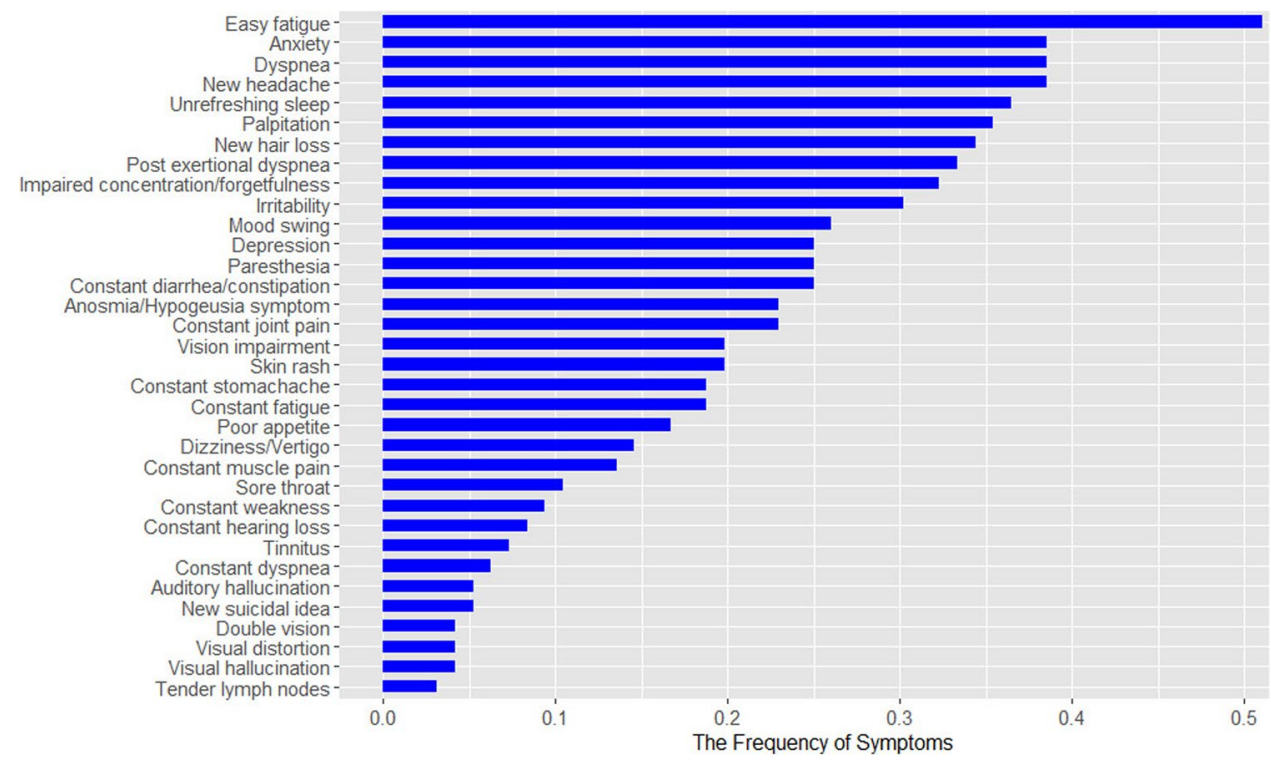

Fig. 2 The frequency of clusters of long-COVID symptoms in the follow-up of 95 patients with a history of COVID-19

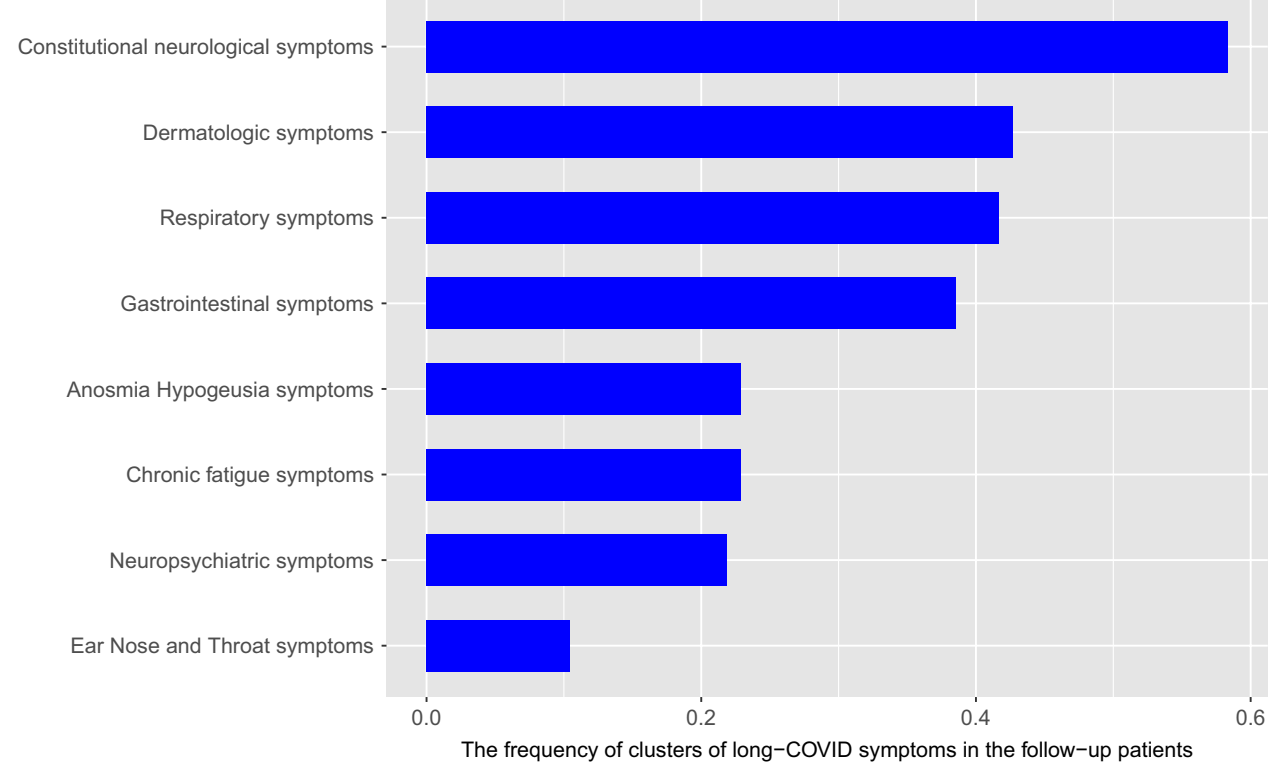

force reduction), olfactory (anosmia and hypogeusia), constitutional cluster (fever and weakness), specific neuropsychiatric (including photophobia, mental state change, hallucination, vision and speech problems, seizures, stroke, and balance disturbance), gastrointestinal (diarrhea and nausea), and respiratory (cough and dyspnea). In the current study, we explored potential associations between long-COVID symptom clusters and these acute-phase symptom clusters. To this end, multiple regression analyses were used to test if the acute-phase COVID symptoms could predict each of the new clusters or symptoms present during the 9-month follow-up. The results of the general linear regression model indicated that the constitutional neuropsychiatric symptoms in the acute phase predicted chronic fatigue syndrome in the follow-up $\left(R^{2}=6.4 \%\right.$, $F(1,93)=2.529, p=0.01)$. Further regression analyses showed that specific neuropsychiatric symptoms in the acute phase were associated with gastrointestinal symptoms $\left(R^{2}=6 \%, F(1,93)=2.43, p=0.01\right)$ in the follow-up. Having specific neuropsychiatric symptoms in the acute phase was also a predictor of dermatologic disturbances in the future $\left(R^{2}=8.5 \%, F(1,93)=2.96, p=0.003\right)$. Moreover, specific neuropsychiatric symptoms predicted respiratory symptoms as long-COVID $\left(R^{2}=9.8 \%, F(1,93)=3.19\right.$, $p=0.001)$. Respiratory symptoms in the acute phase predicted future anosmia and dysgeusia symptoms, and they also marginally predicted olfactory symptoms in the acute phase of COVID-19. Finally constitutional and 


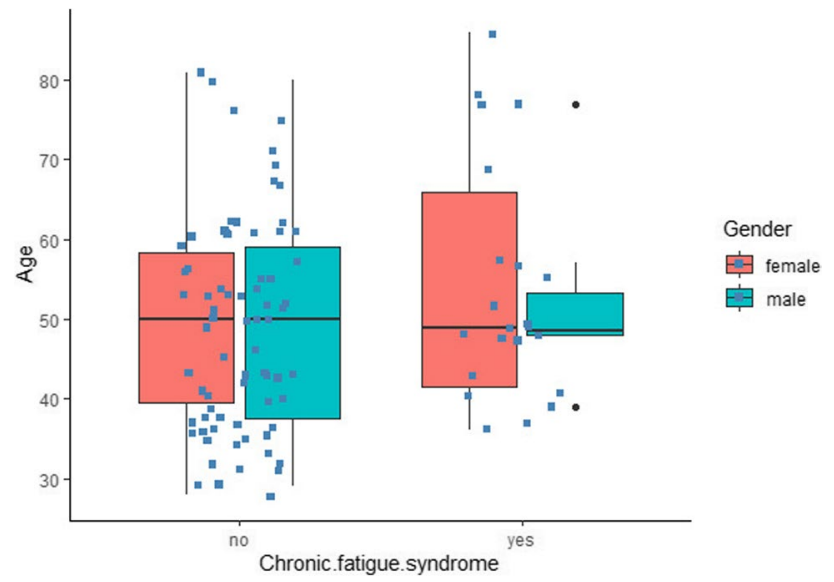

Fig. 3 The relation of chronic fatigue syndrome (CFS) and age/gender

olfactory symptoms in the acute phase were predictors of ear-nose-throat symptoms as long-COVID $\left(R^{2}=16 \%\right.$, $F(2,93)=-1.913, p=0.5, F(2,93)=3.62, p=0.0004$, respectively).

There was no significant association between COVID-19 severity in the acute phase and the number of long-COVID symptoms $(F(1,93)=0.75, p=0.45)$. There was also no significant association between COVID-19 severity in the acute phase and having chronic fatigue syndrome in the future as a long-COVID symptom $(F(1,93)=-0.49, p=0.62)$. However, the number of symptoms in the acute phase could predict having chronic fatigue syndrome as long-COVID $(F(1,93)=2.76, p=0.0068)$, as shown in Fig. 4 .

\section{Cognitive status in the follow-up of patients with COVID-19}

When testing the association between MoCA score and severity of COVID-19, the regression analysis did not show any correlation between MoCA score in the follow-up (cognitive status) and acute-phase disease severity (admission history) of patients with COVID-19 $(F(1,90)=0.073$, $p=0.787$ ). However, it was shown that the duration of COVID-19 was associated with a lower MoCA score, though confounded by the effect of age $(F(1,86)=2.54, p=0.11$, $F(2,85)=26.04, p=1.5 \mathrm{e}-9)$. Furthermore, the number of symptoms in the acute phase did not predict later MoCA scores $\left(R^{2}=6.8 \mathrm{e}-5 \%, F(1,92)=0.079, p=0.937\right)$. ANOVA analysis showed that among the six symptoms clusters at the acute phase of COVID-19, constitutional neuropsychiatric symptoms were associated with lower MoCA scores $(F(1,93)=7.17, p=0.008)$ as you can see in Fig. 5 and they also had poor performance on delayed recall $(F(1,93)=8.91$, $p=0.003$ ).
Fig. 4 The relation of chronic fatigue syndrome (CFS) and the number of symptoms in the acute phase of COVID-19

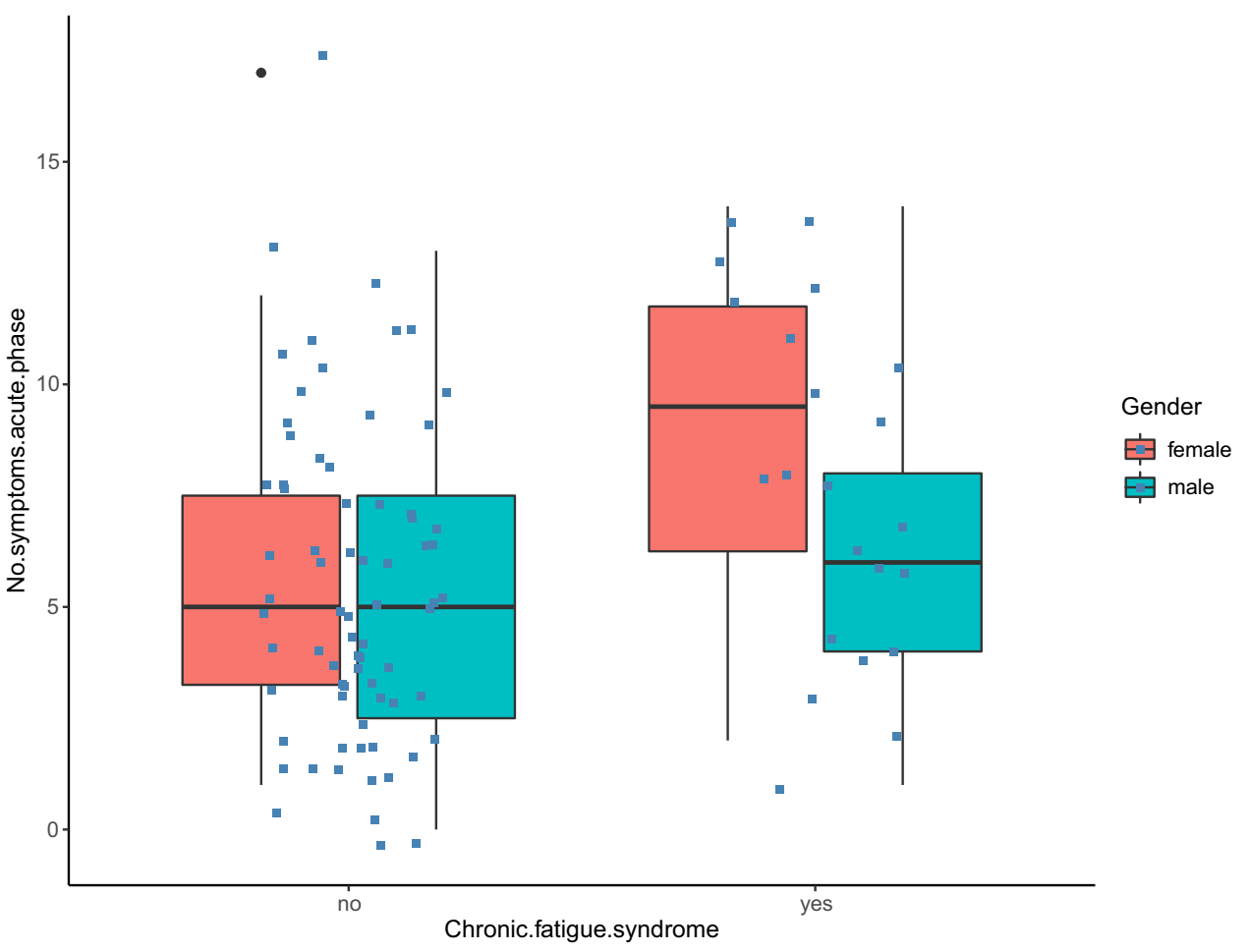


Fig. 5 The relation between the constitutional neuropsychiatric symptoms and follow-up MoCA score

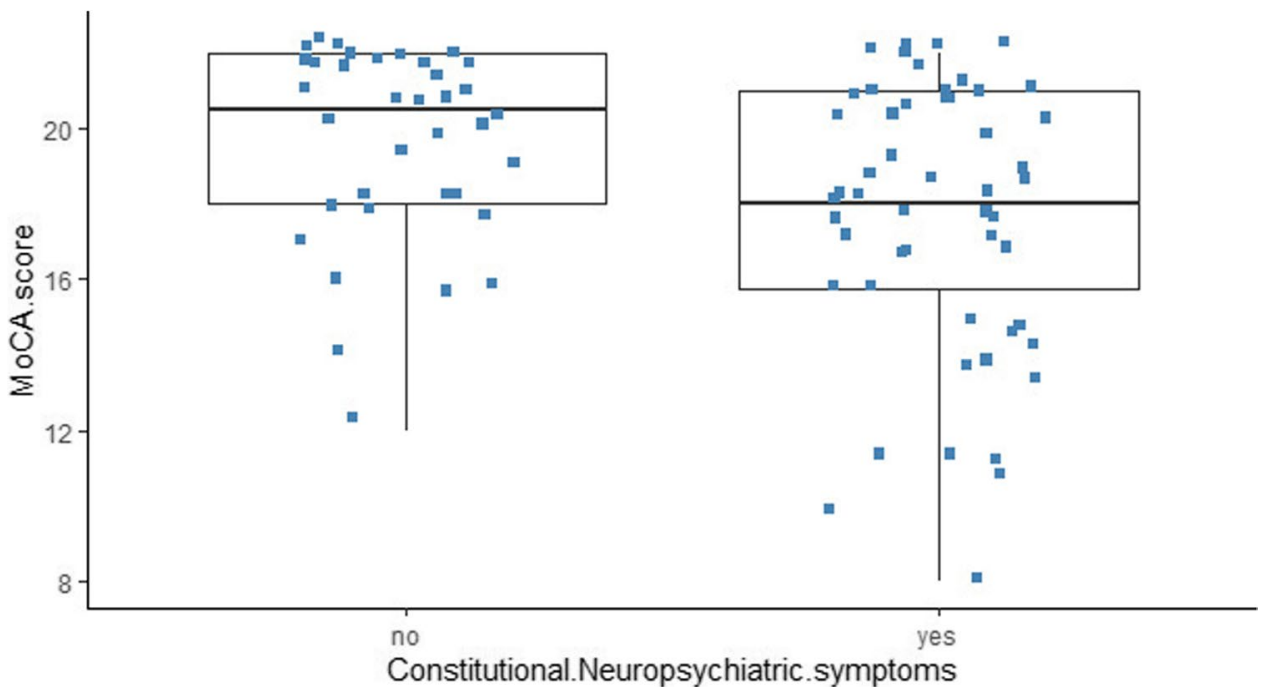

\section{Discussion}

Our study is one of the few attempts to follow-up patients with COVID-19 for several months after the acute phase [18]. We found that there was an association between acute-phase clinical manifestations of COVID-19 and later long-COVID symptoms and cognitive sequelae, although the severity of acute-phase symptoms was not significantly associated with those long-term consequences.

Long-COVID symptoms were very common among our patients: easy fatigability, anxiety, dyspnea, and new-onset headache were the most frequently reported. These findings are consistent with previous research reporting a high prevalence of long-COVID symptoms at follow-up: Sykes et al. reported long-COVID symptoms in $86 \%$ of their patients [25]. Consistent with other studies [26], there was no statistically significant association between disease severity in the acute phase and the risk of developing long-COVID at follow-up. While most previous literature followed COVID-19 patients for a maximum of 6 months [2, 25, 27], our results indicate the presence of persistent symptoms up to 9 months.

Experiencing physical symptoms alongside psychological and cognitive symptoms for several months could be impairing for COVID-19 survivors and may reflect the bio-psychosocial nature of this disease, which supports the need for multidisciplinary rehabilitation approaches. Previous literature has suggested that long-COVID represents a miscellany of physical and psychological symptoms, which, like Persian Gulf War illness, could even include a form of post-traumatic disorder [25]. We found that there was an association between the symptom clusters in the acute-phase and longCOVID symptoms and they were not just random miscellany of symptoms coming together, which makes long-COVID as a sole post-traumatic stress syndrome less probable [28]. We found that the constitutional neuropsychiatric symptom cluster was associated with having a lower MoCA score at follow-up, specifically more impaired delayed recall, while the number of COVID-19 symptoms in the acute phase, the severity of COVID-19 presented by the status of admission, and the duration of COVID-19 symptoms in the acute phase were not. These findings show that the pattern of organ involvement in COVID-19, specifically in the acute phase, could predict how patients would experience longCOVID in the future. It seems that systemic inflammation, even when it is not severe, could lead to cognitive impairment in the future if it involves neuroinvasion in the acute phase $[29,30]$. These findings are also comparable with those of Guedj et al., who found that patients with longCOVID showed hypometabolism patterns in different brain regions such as bilateral rectal/orbital gyrus, right temporal lobe, and amygdala, which were associated with memory and cognitive impairment [31]. Those findings further confirm that long-COVID symptoms are more than a random miscellany and suggest that they may be associated with disbalance in brain networks. Our findings are also consistent with previous studies which reported an age-dependent cognitive decline [32] apathy, executive dysfunction, reduction in global cognition along with GABAergic disbalance as the underlying mechanisms for executive dysfunction in the long term for COVID-19 patients [33].

We also found that specific neuropsychiatric involvement in the acute phase was a good predictor of long-COVID in different organs in the follow-up. Experiencing specific neuropsychiatric symptoms in the acute phase was associated with multisystem involvement such as specific gastrointestinal, dermatologic, and respiratory symptoms in the follow-up. Sollini et al. showed that vascular inflammation could be the leading cause of long-lasting COVID symptoms [28]. However, anosmia and dysgeusia in the acute phase of COVID-19 were associated with ear-throat-nose or inner ear (tinnitus and hearing 
loss) symptoms at follow-up. It is not yet clear whether these organs share a similar receptor in their vasculature or other cellular structure. Persistent endotheliopathy has been manifest in convalescent COVID-19 and as a probable underlying mechanism of long-COVID [34].

Being female, experiencing constitutional neuropsychiatric symptom categories in the acute phase, and having a higher number of symptoms in the acute phase could predict later chronic fatigue syndrome. This was consistent with other results in our study showing that the severity of the COVID-19 in the acute phase could not predict developing long-COVID in the future. It seems that it is also true for chronic fatigue syndrome and what is more important to predict the risk of chronic fatigue syndrome in the future is the pattern of organ involvement in the acute phase rather than the severity of the disease (low saturation, loss of consciousness, shock, admission status). This suggests that the mechanism of chronic fatigue syndrome is similar to other long-COVID symptoms, which is consistent with previous research findings [35]. Townsend et al. also found COVID-persistent fatigue to be more common in females and not associated with acute illness severity (admission status and laboratory inflammatory markers) [35]. One possible mechanism would be a state of chronic low-grade neuroinflammation followed by the disease [29]. COVID-19 can infect the brain leading to neuroinflammation [36]. Alternatively, it can cause inflammation in other parts of the body, activating an innate immune response in the brain via humoral response and retrograde vagus nerve transmission [30, 37]. Neuroinflammation can cause severe fatigue [38]. This would explain the association of constitutional neuropsychiatric symptoms in the acute phase and chronic fatigue syndrome in the future.

Some important limitations of our study should be highlighted. A large number of lost to follow-up patients may have induced a selection bias. Face-to-face full-depth interviews and examinations might be a more accurate measurement of the patients' symptoms compared to telephone follow-up. Only a small number of patients had full workup of paraclinical data and chest imaging in the electronic system of the hospital; therefore, analyses of the association between those data and long-COVID symptoms were not carried out. Future studies to explore the association between symptom clusters and inflammatory markers in the acute-phase and long-COVID symptoms could shed new light on the underlying mechanism of the post-acute phase in people suffering from COVID-19.

\section{Conclusion}

Most of our patients experienced at least one physical, psychological, or cognitive symptom within 9 months following acute COVID-19 infection. Easy fatigability, followed by headache, anxiety, and dyspnea were the most common long-COVID symptoms. There was an association between the acute presentation of COVID-19 and long-COVID symptoms in the follow-up, which was not related to disease severity. Having constitutional neuropsychiatric symptoms in the acute phase was associated with chronic fatigue syndrome, lower MoCA score, and poorer performance on delayed recall in the future. Having specific neuropsychiatric symptoms in the acute phase was associated with multisystem involvement such as dermatologic, gastrointestinal, and respiratory symptoms as long-COVID. Olfactory symptoms in the acute phase were associated with inner ear complaints in the future. These results provide further insight into the long-lasting physical and neuropsychiatric sequelae of COVID-19 and may contribute to the understanding of their physiopathology and the study of short-term treatments and long-term rehabilitation approaches for this disease.

Acknowledgements The authors wish to thank Rasool Akram Medical Complex Clinical Research Development Center (RCRDC), Iran University of Medical Science, for technically supported implementation of project.

\section{Declarations}

Ethical approval This research has been approved by the Ethics Committee of Iran University of Medical Science (code number: IR.IUMS. REC.1399.1040). The privacy rights of human subjects must always be observed. All principles of the latest version of Helsinki's declaration on ethical principles of human studies were met through all the study steps.

Informed consent All of the patients gave informed consent for the study.

Conflict of interest None.

\section{References}

1. Worldometers (2021) https://www.worldometers.info/coronavirus/. 2021

2. Nalbandian A, Sehgal K, Gupta A, Madhavan MV, McGroder C, Stevens JS, Cook JR, Nordvig AS, Shalev D, Sehrawat TS, Ahluwalia N (2021) Post-acute COVID-19 syndrome. Nature Med 27(4):601-615

3. Greenhalgh T, Knight M, Buxton M, Husain L (2020) Management of post-acute covid-19 in primary care. BMJ 370:m3026

4. Mahase E (2021) Covid-19: what do we know about "long covid"? BMJ 370

5. Carvalho-Schneider C, Laurent E, Lemaignen A, Beaufils E, Bourbao-Tournois C, Laribi S, Flament T, Ferreira-Maldent N, Bruyère F, Stefic K, Gaudy-Graffin C (2021) Follow-up of adults with noncritical COVID-19 two months after symptom onset. Clin Microbiol Infect 27(2):258-263

6. Huang C, Huang L, Wang Y, Li X, Ren L, Gu X, Kang L, Guo L, Liu M, Zhou X, Luo J (2021) 6-month consequences of COVID19 in patients discharged from hospital: a cohort study. Lancet 397(10270):220-232 
7. Nakamura ZM, Nash RP, Laughon SL, Rosenstein DL (2021) Neuropsychiatric complications of COVID-19. Curr Psychiatr Rep 23(5):1-9

8. Mazza MG, De Lorenzo R, Conte C, Poletti S, Vai B, Bollettini I, Melloni EM, Furlan R, Ciceri F, Rovere-Querini P, Benedetti F (2020) Anxiety and depression in COVID-19 survivors: Role of inflammatory and clinical predictors. Brain Behav Immunol 89:594-600

9. Deng J, Zhou F, Hou W, Silver Z, Wong CY, Chang O, Huang E, Zuo QK (2021) The prevalence of depression, anxiety, and sleep disturbances in COVID-19 patients: a meta-analysis. Ann New York Acad Sci 1486(1):90-111

10. Sudre CH, Lee KA, Lochlainn MN, Varsavsky T, Murray B, Graham MS, Menni C, Modat M, Bowyer RC, Nguyen LH, Drew DA (2021) Symptom clusters in COVID-19: a potential clinical prediction tool from the COVID Symptom Study app. Sci Adv 7(12):eabd4177

11. Garrigues E, Janvier P, Kherabi Y, Le Bot A, Hamon A, Gouze H, Doucet L, Berkani S, Oliosi E, Mallart E, Corre F (2020) Post-discharge persistent symptoms and health-related quality of life after hospitalization for COVID-19. J Infect 81(6):e4-6

12 Ritchie K, Chan D, Watermeyer T (2020) The cognitive consequences of the COVID-19 epidemic: collateral damage? Brain Commun 2(2):fcaa069

13. Taquet M, Luciano S, Geddes JR, Harrison PJ (2021) Bidirectional associations between COVID-19 and psychiatric disorder: retrospective cohort studies of 62354 COVID-19 cases in the USA. Lancet Psychiatry 8(2):130-140

14. Woo MS, Malsy J, Pöttgen J, SeddiqZai S, Ufer F, Hadjilaou A, Schmiedel S, Addo MM, Gerloff C, Heesen C, Schulze ZurWiesch J (2020) Frequent neurocognitive deficits after recovery from mild COVID-19. Brain Commun 2(2):fcaa205

15. Kennedy M, Helfand BK, Gou RY, Gartaganis SL, Webb M, Moccia JM, Bruursema SN, Dokic B, McCulloch B, Ring H, Margolin JD (2020) Delirium in older patients with COVID-19 presenting to the emergency department. JAMA Netw Open 3(11):e2029540

16. Pun BT, Badenes R, La Calle GH, Orun OM, Chen W, Raman R, Simpson BG, Wilson-Linville S, Olmedillo BH, de la Cueva AV, van der Jagt M (2021) Prevalence and risk factors for delirium in critically ill patients with COVID-19 (COVID-D): a multicentre cohort study. Lancet Respir Med 9(3):239-250

17. Iqbal A, Iqbal K, Ali SA, Azim D, Farid E, Baig MD, ... Raza M (2021) The COVID-19 sequelae: a cross-sectional evaluation of post-recovery symptoms and the need for rehabilitation of COVID-19 survivors. Cureus 13(2)

18. Mirfazeli FS, Sarabi-Jamab A, Jahanbakhshi A, Kordi A, Javadnia P, Shariat SV, Aloosh O, Almasi-Dooghaee M, Faiz SH (2020) Neuropsychiatric manifestations of COVID-19 can be clustered in three distinct symptom categories. Sci Rep 10(1): $1-9$

19. World Health Organization (2020) Clinical management of severe acute respiratory infection (ISARI)" when COVID-19 disease is suspected: interim guidance, 13 March 2020 (No. WHO/2019-nCoV/clinical/2020.4). World Health Organization

20. Fukuda K, Straus SE, Hickie I, Sharpe MC, Dobbins JG, Komaroff A (1994) The chronic fatigue syndrome: a comprehensive approach to its definition and study. Ann Intern Med 121(12):953-959

21. Nasreddine ZS, Phillips NA, Bédirian V, Charbonneau S, Whitehead V, Collin I, Cummings JL, Chertkow H (2005) The Montreal Cognitive Assessment, MoCA: a brief screening tool for mild cognitive impairment. J Am Geriatr Soc 53(4):695-699

22. Iiboshi K, Yoshida K, Yamaoka Y, Eguchi Y, Sato D, Kishimoto M, Funaki K, Mimura M, Kishimoto T (2020) A validation study of the remotely administered Montreal Cognitive
Assessment tool in the elderly Japanese population. Telemed E-Health 26(7):920-928

23. DeYoung N, Shenal BV (2019) The reliability of the Montreal Cognitive Assessment using telehealth in a rural setting with veterans. J Telemed Telecare 25(4):197-203

24. Wong A, Nyenhuis D, Black SE, Law LS, Lo ES, Kwan PW, Au L, Chan AY, Wong LK, Nasreddine Z, Mok V (2015) Montreal Cognitive Assessment 5-minute protocol is a brief, valid, reliable, and feasible cognitive screen for telephone administration. Stroke 46(4): 1059-1064

25. Sykes DL, Holdsworth L, Jawad N, Gunasekera P, Morice AH, Crooks MG (2021) Post-COVID-19 symptom burden: what is long-COVID and how should we manage it? Lung 199(2):113-119

26. Mandal S, Barnett J, Brill SE, Brown JS, Denneny EK, Hare SS, Heightman M, Hillman TE, Jacob J, Jarvis HC, Lipman MC (2021) 'Long-COVID': a cross-sectional study of persisting symptoms, biomarker and imaging abnormalities following hospitalisation for COVID-19. Thorax 76(4):396-398

27. Sudre CH, Murray B, Varsavsky T, Graham MS, Penfold RS, Bowyer RC, Pujol JC, Klaser K, Antonelli M, Canas LS, Molteni E (2020) Attributes and predictors of Long-COVID: analysis of COVID cases and their symptoms collected by the Covid Symptoms Study App. Medrxiv

28. Sollini M, Ciccarelli M, Cecconi M, Aghemo A, Morelli P, Gelardi F, Chiti A (2021) Vasculitis changes in COVID-19 survivors with persistent symptoms: an [18 F] FDG-PET/CT study. Eur J Nucl Med Mol Imaging 48(5):1460-1466

29. Mueller C, Lin JC, Sheriff S, Maudsley AA, Younger JW (2020) Evidence of widespread metabolite abnormalities in Myalgic encephalomyelitis/chronic fatigue syndrome: assessment with whole-brain magnetic resonance spectroscopy. Brain Imaging Behav 14(2):562-572

30. Poon DC, Ho YS, Chiu K, Wong HL, Chang RC (2015) Sickness: from the focus on cytokines, prostaglandins, and complement factors to the perspectives of neurons. Neurosci Biobehav Rev 57:30-45

31. Guedj E, Campion JY, Dudouet P, Kaphan E, Bregeon F, TissotDupont H, Guis S, Barthelemy F, Habert P, Ceccaldi M, Million M (2021) 18F-FDG brain PET hypometabolism in patients with long COVID. Eur J Nucl Med Mol Imaging 26:1-11

32. Alemanno F, Houdayer E, Parma A, Spina A, Del Forno A, Scatolini A, Angelone S, Brugliera L, Tettamanti A, Beretta L, Iannaccone S (2021) COVID-19 cognitive deficits after respiratory assistance in the subacute phase: a COVID-rehabilitation unit experience. Plos One 16(2): 0246590

33. Ortelli P, Ferrazzoli D, Sebastianelli L, Engl M, Romanello R, Nardone R, Bonini I, Koch G, Saltuari L, Quartarone A, Oliviero A (2021) Neuropsychological and neurophysiological correlates of fatigue in post-acute patients with neurological manifestations of COVID-19: Insights into a challenging symptom. J Neurol Sci 420:117271

34. Fogarty H, Townsend L, Morrin H, Ahmad A, Comerford C, Karampini E et al (2021) Persistent endotheliopathy in the pathogenesis of long COVID syndrome. J Thromb Haemost 19(10):2546-2553

35. Townsend L, Dyer AH, Jones K, Dunne J, Mooney A, Gaffney F, O'Connor L, Leavy D, O'Brien K, Dowds J, Sugrue JA (2020) Persistent fatigue following SARS-CoV-2 infection is common and independent of severity of initial infection. Plos One 15(11):e0240784

36. Pan R, Zhang Q, Anthony SM, Zhou Y, Zou X, Cassell M, Perlman S (2020) Oligodendrocytes that survive acute coronavirus infection induce prolonged inflammatory responses in the CNS. Proc Natl Acad Sci 117(27):15902-15910 
37. Van Elzakker MB (2013) Chronic fatigue syndrome from vagus nerve infection: a psychoneuroimmunological hypothesis. Med Hypotheses 81(3):414-423

38. Dantzer R, Heijnen CJ, Kavelaars A, Laye S, Capuron L (2014)

The neuroimmune basis of fatigue. Trends Neurosc 37(1):39-46
Publisher's note Springer Nature remains neutral with regard to jurisdictional claims in published maps and institutional affiliations.

\section{Authors and Affiliations}

\section{Fatemeh Sadat Mirfazeli ${ }^{1}$ (D) - Atiye Sarabi-Jamab ${ }^{2}$ (D) . Victor Pereira-Sanchez ${ }^{3,4} \mathbb{D} \cdot$ Alireza Kordi $^{5}$ (D) Behnam Shariati $^{1}$ (D) S Seyed Vahid Shariat ${ }^{1,6}$ (D) Salar Bahrami ${ }^{5}$ (D) . Shabnam Nohesara ${ }^{1}$. \\ Mostafa Almasi-Dooghaee ${ }^{7}$ (D) Seyed Hamid Reza Faiz ${ }^{8}$ (D)}

Fatemeh Sadat Mirfazeli

Mirfazeli.f@iums.ac.ir

Atiye Sarabi-Jamab

a.sarabi@ipm.ir

Victor Pereira-Sanchez

vpereira@alumni.unav.es

Alireza Kord

alirezakordi2010@yahoo.com

Behnam Shariati

Shariati.b@iums.ac.ir

Seyed Vahid Shariat

shariat.v@iums.ac.ir

Salar Bahrami

b.salar75@outlook.com

Shabnam Nohesara

shnohesara@gmail.com

Mostafa Almasi-Dooghaee

Almasidoghaei.m@iums.ac.ir

1 Mental Health Research Center, Psychosocial Health Research Institute (PHRI), Department of Psychiatry, School of Medicine, Iran University of Medical Sciences, Tehran, Iran
2 School of Cognitive Sciences, Institute for Research in Fundamental Sciences (IPM), P. O. Box 19395-5746, Tehran, Iran

3 Department of Psychiatry, Columbia University, New York, NY, USA

4 Department of Child and Adolescent Psychiatry, NYU Grossman School of Medicine, New York, NY, USA

5 School of Medicine, Iran University of Medical Sciences, Tehran, Iran

6 Mental Health Research Center, Psychosocial Health Research Institute, Department of Psychiatry, School of Medicine, Iran University of Medical Sciences, Tehran, Iran

7 Neurology Department, Firoozgar hospital, School of Medicine, Iran University of Medical Sciences, Tehran, Iran

8 Department of Anesthesiology and Pain Medicine, Minimally Invasive Surgery Research Center, Iran University of Medical Sciences, Rasool Akram Medical Complex, Sattarkhan St, Tehran, Iran 